\title{
Mobile Sink-based Self Adaptive and Energy Efficient Proactive Data Collection Protocol for Wireless Sensor Network
}

\author{
Dheeraj D. Umardand \\ P. G. Student, \\ MBES College of Engineering, \\ Ambajogai, India, 431517.
}

\author{
Chirchi V. R. \\ P. G. Dept. \\ MBES College of Engineering, \\ Ambajogai, India, 431517.
}

\begin{abstract}
In Wireless Sensor Networks (WSNs), trailing data sinks' mobility for data gathering has drawn popular in recent years. To achieve optimized network performance, or target at collecting a small portion of sensed data in the network recent researches will either focus on planning a mobile sink's moving trajectory in advance. In many application scenarios, however, a mobile sink cannot move freely in the deployed area. In this paper they propose energy consuming proactive data reporting protocols, SinkTrail for mobile sink-based data collection to avoid constant sink location update traffics when a sink's future locations cannot be scheduled in advance. SinkTrail differs their approach from previous ones: 1) allow sufficient flexibility in the movement of mobile sinks and also predict various terrestrial changes. 2) SinkTrail doesn't require GPS devices or predefined anchors (landmarks); it establishes a logical coordinate system for routing and forwarding data packets, making it suitable for various application scenarios. They systematically observed the impact of several design factors in the proposed algorithms. Finally conclude that both theoretical analysis and simulation results demonstrate that the proposed algorithms reduce control overheads and good performance in finding shorter routing paths.
\end{abstract}

\section{Keywords}

Wireless sensor network, Sink, Routing, GPS, and Mobile sink.

\section{INTRODUCTION}

Wireless Sensor Network (WSN) is a type of wireless network, consists of a collection of sensor nodes. Sensor node performs following tasks; (i) sample a physical quantity from the surrounding environment, (ii) process (and possibly store) the acquire data, and (iii) transfer them through wireless communications to a data collection point called sink node or base station. Wireless sensor network with sensor nodes work together to detect a region to collect data about environment. Typical WSNs are composed of a large number of sensor nodes which transmit the sensed information to the sink. Since a sensor node is constrained by a device with limited power supply, recharging sensor nodes is often infeasible. One of the most important challenges in large-scale WSNs is energy efficient algorithms, since sensor nodes have restricted energy. For example, if some sensor nodes fail due to insufficient power, then WSNs may not fulfill their functions properly. Therefore, less energy consumption of sensor nodes and maximize the lifetime of the entire network have significant importance in the design of sensor network protocols. So focus on data collection concludes that allowing and leveraging sink mobility is more challenge full for energy efficient data gathering rather than reporting data through long, multihop count routes to a static sink. So using mobile sinks data gathering becomes new challenges to sensor network applications. Studying or scheduling movement patterns of a mobile sink to visit some special places in a deployed area in order to minimize data gathering time. In such cases a mobile sink moves to predetermined trail points and ask about data report to each sensor node individually. In this paper, they introduce SinkTrail protocol which is selfpredictable and proactive data reporting protocol for various application areas. In this protocol mobile sinks move in the deployed area continuously with low speed, and gather data. From existing data gathering protocols they introduced some Control messages that are broadcasted in much lower frequency. In SinkTrail, mobile sinks move continuously in the field in relatively low speed, and collect data on the fly. Control messages are broadcasted at certain points in much lower frequency than ordinarily required in existing data gathering protocols. These known positions are viewed as "footprints" of a mobile sink. Considering each footprint as a virtual landmark, a sensor node can easily identify its hop count distances to these landmarks. These hop count distances combined represent the sensor node's coordinate in the logical coordinate space constructed by the mobile sink. Similarly, the coordinate of the mobile sink is its hop count distances from the current location to previous virtual known points. Having the destination coordinate and its own coordinate, each sensor node greedily selects next hop with the shortest logical distance to the mobile sink. As a result, SinkTrail solves the problem of movement prediction for data gathering with mobile sinks. In this paper they contribute the following feature.

- Paper determine a unique logical coordinate representation system for mobile sinks without using GPS devices or predefined locations, which is widely applicable to various scenarios.

- It also define dynamic routing protocol for data gathering with one or multiple mobile sink(s), which minimizes average route length and reduces total energy consumption.

- Paper conducts extensive comparison studies and simulations with popular existing solutions.

The rest of the paper is organized as follows. Section 2 presents related work. Detailed protocol design is introduced in Section 3. Section 4 presents analytical and simulation results, and demonstrates the advantages of SinkTrail, The impact of several simulated parameter of sensor network on SinkTrail is investigated and analyzed. Section 5 concludes the paper. 


\section{LITERATURE SURVEY}

Data sinks mobility is the most challenging part in sensor data collection which is to be effectively handling the control overheads introduced by a sink's movement. At the first look, multicasting is the most natural solution to track the moving mobile sink. This approach is sink oriented and in previous research efforts, e.g., [1], [4], [13], have demonstrated its effectiveness in collecting a small amount of data from the network [17]. To reduce control messages they had following protocols, TTDD protocol, proposed in [12], constructed a two-tier data dissemination structure in advance to enable fast data forwarding [17]. In [3], a spatialtemporal multicast protocol is proposed to establish a delivery zone ahead of mobile sink's arrival [17]. Similarly, Park et al. [9] proposed DRMOS that divides sensors into "wake-up" zones to save energy [17]. Luo and Hubaux [7] proposed that a mobile sink should move following a circle trail in deployed sensor field to maximize data gathering efficiency [17].Disadvantage of the multicasting method is its flooding nature. Let us consider that mobile sinks move at a fixed velocity and fixed direction, or follow a fixed moving pattern, which largely confines their application. Another category of methods, called Mobile Element Scheduling (MES) algorithms [2], [8], [10], [11], [14], [15], [16], considered controlled mobile sink mobility and advanced planning of mobile sink's moving path [17]. Ma and Yang [8] focused on minimizing the length of each data gathering tour by intentionally controlling the mobile sink's movement to query every sensor node in the network [17].

Whenever data sampling rates in the network are heterogeneous, scheduling mobile sinks to visit hot-spots of the sensor network becomes helpful. Example algorithms can be found in [2], [11].MES algorithm is useful to reduce data transmission costs and in the sensor field they need a mobile sink to cover every node. Even worse, finding an optimal data gathering tour in general is itself an NP-hard problem [6], [8], and constrained access areas or obstacles in the deployed field pose more complexity [17]. For sink location prediction and selects data reporting routes SinkTrail uses greedy algorithm. In [5], keally et al. used sequential Monte Carlo theory to predict sink locations to enhance data reporting [17]. SinkTrail deploys a different prediction technique that has much lower complexity.

\section{PROPOSED SYSTEM}

Once the data gathering process has been started, the mobile sink moves around in IN keeps listening for data report. Sensor are connected and achieved by deploying deeply. Sensor nodes are awaked by synchronization or wake up massage and then the actual data gathering process starts. To gather data from predetermined IN network, they periodically send out a number of mobile sinks into the field. SinkTrail protocol has been designed mainly into two modules:

\subsection{SinkTrail Protocol working with One Mobile Sink}

The mobile sink moves around in IN keeps listening for data report packets when the data gathering process starts. Let us consider that $\bar{\tau}$ be the average transmission range. In network two adjacent trail points must be separated by a distance longer than $\bar{\tau}$, because the hop counts information will be significantly same. The distances between any two consecutive trail points are same (or similar), denoted as $\mathrm{K} \bar{\tau}$, $\mathrm{K}>1$. A mobile sink contains a trail message which contains sequence number (msg.seqN) and a hop count (msg.hopC) to the sink. In this protocol one "move" means the time interval between a mobile sink stops at one trail point and arrives at the next trail point. There may be multiple moves during a data gathering process. In the SinkTrail algorithm, another parameter use called as vectors i.e. "Trail References" to represent logical coordinates in a network [18]. The trail reference maintained by each node is used for packet forwarding by indicating its location. Following notations are used throughout the protocol description are in Table 1.

Table 1.Different notations used in the protocol description.

\begin{tabular}{|c|l|}
\hline Notation & Description \\
\hline$n_{i}$ & Sensor node I \\
\hline $\mathrm{N}$ & Total number of sensor node in $\mathrm{N}$ \\
\hline $\mathrm{S}$ & Mobile sink \\
\hline $\mathrm{M}$ & Number of mobile sink \\
\hline$e_{i}^{j}$ & Trail reference of node $\mathrm{i}$ \\
\hline$v_{i}$ & The jth element in vi \\
\hline$d_{v}$ & Trail reference size $d_{v}=\|\mathrm{v}\|$ \\
\hline $\mathrm{B}$ & Avg no of neighbors of each node \\
\hline$\lambda$ & $\begin{array}{l}\text { Latest message sequence number a node has } \\
\text { recorded }\end{array}$ \\
\hline$\pi_{i}$ & The ith trail point of s \\
\hline$\pi$ & Collection of trail points \\
\hline$D_{\pi}$ & Total number of trail points \\
\hline $\mathrm{K}$ & Step size parameter for one move \\
\hline
\end{tabular}

All trail references should be of the same size. In this protocol the data reporting procedure consists mainly three phases as follows.

\subsubsection{Virtual Coordinate Identification and \\ Construction}

Whenever the mobile sink $\mathrm{S}$ travel through the Network size $\mathrm{IN}$, all sensor nodes' trail references are initialized to [-1,-1,-

1] of size $d_{v}$ [18].To track the latest message sequence number a special variable $\lambda$ is used that is also set to -1 because initially nodes doesn't have any co-ordinates to know their exact position in the network. After the mobile sink $\mathrm{S}$ enters the field, it stops at some places and randomly selects a

place as its first trail point $\pi_{1}$, and broadcasts a trail message to all the sensor nodes in IN to identify exact position from the nodes. The trail message contains, <msg.seqN, msg:hopC $\rangle$, is set to $\langle 1,0\rangle$, which indicates that this is the first trail message from trail point one, and the hop count to $S$ is zero. The nodes nearest to $S$ will be act as neighbor and hear this message first. To check the freshness of message a node needs to be compare with $\lambda$, if this is a latest message, then $\lambda$ will be updated with the latest new sequence number. And node $n_{i}$ 's trail reference $v_{i}$ is updated as follows: first, every element in $v_{i}$ is shifted to left by one position. Then the hop count in the received trail message is increased by one, and replaces the right-most element $e_{i}^{d v}$ in $v_{i}$. After $n_{i}$ updated its trail reference, this trail message is rebroadcasted with the same sequence number and an incremented hop count [17]. The same procedure repeats at all 
the other nodes in IN. Within one move of S, all nodes in the network have updated their trail references according to their hop count distances to S's trail point $\pi_{1}$.Following Figure.1 shows overall module structure of SinkTrail protocol.

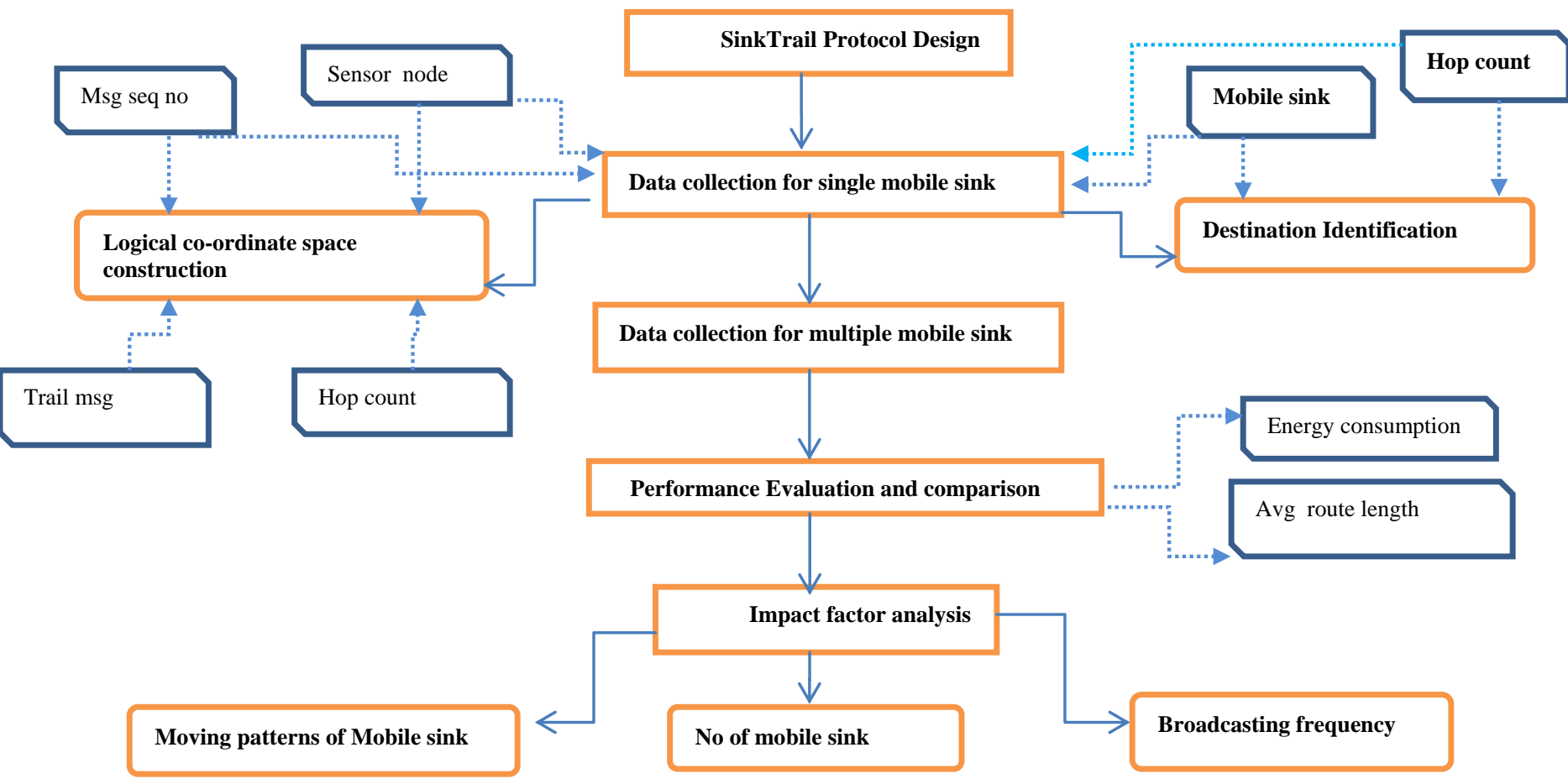

Figure 1. Abstract model diagram of SinkTrail.

If a node receives a trail message with sequence number equals to $\lambda$, but has a smaller hop count than it has already recorded, then the last hop count field in its trail reference is updated, and this trail message is rebroadcasted with the same sequence number and an incremented hop count. Trail message that has sequence number less than $\lambda$ will be discarded to eliminate flooding messages in the network.

\section{Wireless Sensor Network}

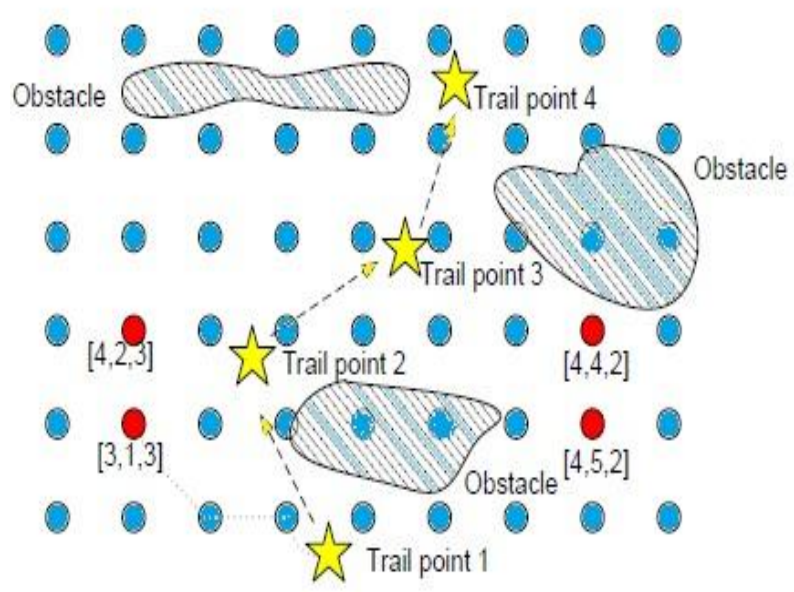

Figure 2. Data gathering process with one mobile sink: yellow stars indicate the mobile sink's trail points, and red sensor nodes maintain trail references as logical coordinates.
During the data gathering procedure, a node's trail reference needs to be updated every time a new trail message is received. After each node in the network received dv distinct trail messages, the logical coordinate space is established. A snapshot of a part of the network IN is shown in Figure.2 Trail references, such as $[3,1,1]$ or $[2,2,2]$, are considered logical coordinates of the sensor nodes in a network [1].

\subsubsection{Destination Reference Identification}

The mobile sink will goes through the some locations according to current field situations. These locations of a mobile sink, named trail points in SinkTrail, are footprints left by a mobile sink, and they provide valuable information for tracing the current location of a mobile sink [17].SinkTrail has the feature like logical coordinate of a mobile sink that keeps changes at each trail point, given the continuous update of trail references to each node in the network. This is because the mobile sink's hop count distance to its previous $d_{\mathrm{v}}-1$ footprints are always $K\left(d_{w}-1\right), K\left(d_{v}-2\right), K$, and 0 to its current location. Therefore, the logical coordinate $\left[K\left(d_{v}-1\right)\right.$, $\left.\mathrm{K}\left(d_{v}-2\right), 0\right]$ represents the current logical location of the mobile sink [17]. 


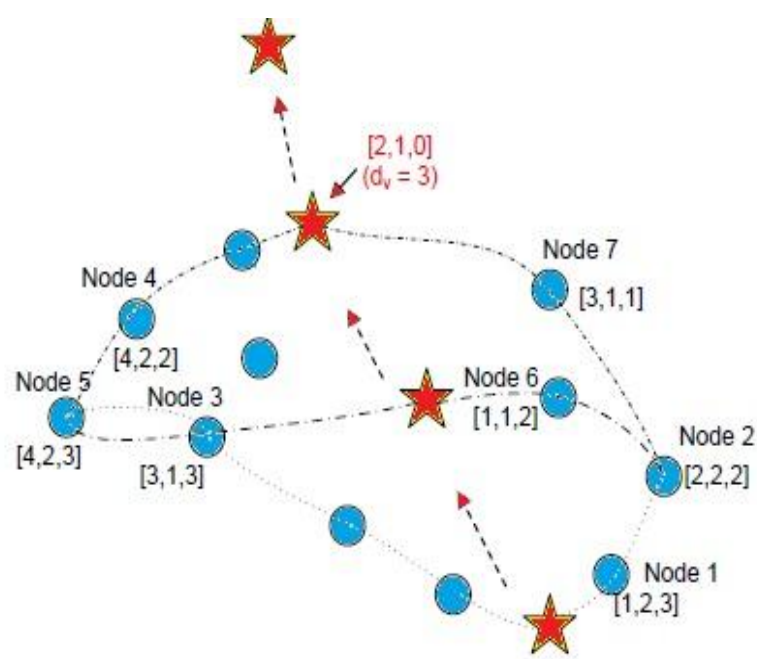

Figure 3. SinkTrail: red stars indicate trail points, and directed arrows stand for the moving path of mobile sink.

Such a coordinate called as "Destination Reference." This destination reference does not necessarily require a mobile sink to have linear moving trajectory. Although arbitrary movement of a mobile sink may deteriorate the accuracy of destination reference, it can still serve as a guideline for data reporting [17]. Here, set $\mathrm{K}=1$ and $=3$ to ease our presentation. Whenever there is less broadcast frequency a value of $\mathrm{K}$ is large. In Figure.3, assume $\mathrm{S}$ moves at the trail Point 3 now, and then its destination reference should be updated $[2,1,0]$ from above formula. When $\mathrm{S}$ moves to the trail Point 4 , the coordinate space is also updated based on trail points 2,3 , and 4 , and destination reference of the mobile sink is still $[2,1,0]$.

\subsubsection{Greedy Forwarding for Data Reporting}

A node has updated the three elements in its trail reference (assume $d_{v}=3$ ), it starts a timer that is inverse proportional to the hop count element in its trail reference. For example, node $n_{5}$ 's trail reference is $[6,7,9]$ in Fig.2, then the duration of its timer is set to $T_{5}=T_{\text {init }}-\mu * 9$.Here, $T_{\text {init }}$ and $\mu$ are predefined constants and they may vary according to timer. In this algorithm timer durations are longer than the trail message's propagation time, so all nodes are viewed as same timer starting at the same time. As soon as node's timer expires, it continues the data reporting process. Timer mechanism is mainly used to differ data reporting orders from mobile sink to nodes and vice versa. Each sensor node in the network maintains a routing table of all neighbour's trail references. Every time as soon as the mobile sink updates its location, routing table is also updated by exchanging trail references with neighbours. When a node has received all its neighbour's trail references, it calculates their distances to the destination reference, $[2,1,0]$, according to 2 -norm vector calculation, and greedily choose the node with the smallest distance as next hop to relay data. If both nodes have same hop count then randomly choose one. Take the network in Figure. 3 as an example, when node $n 5$ decides to report its data, it compares $\mathrm{n} 3$ and $\mathrm{n} 4$ 's vector distance with $[2,1,0]$. Since $n 3$ 's distance to $[2,1,0]$ is $\sqrt{ } 10$, and $n 4$ 's distance is 3 , $\mathrm{n} 4$ is chosen as the next hop of $\mathrm{n} 5$.

\subsection{SinkTrail Protocol working with Multiple Mobile Sink}

SinkTrail protocol can be extended to multiple mobile sinks scenario with small modifications because with one mobile sink they can't collect data from each and every node so this process becomes quite hazy. In the network whenever there are more than one sink, each mobile sink broadcasts trail messages. A little bit change from one sink scenario, a sender ID field, msg.sID, is added to each trail message to distinguish them from another senders. A sensor node maintains multiple trail references for multiple mobile sinks. Each corresponds to a different mobile sink at the same time. Fig.4 shows an example of two mobile sinks. Each sensor node has two trail references, colored in black and red, coexist in the same network. So multiple logical coordinate are constructed simultaneously, and each of them is constructed according to trail points of different mobile sinks. Every time a trail message arrives, the sensor node will determine whether the mobile sink's ID in the message is old one then it is considered as old mobile sink else it will create a new trail reference for new mobile sink. In SinkTrail trail references of each node represent node locations in different logical coordinate spaces, when it comes to data forwarding, because reporting to any mobile sink is valid, the node can choose the neighbor closest to a mobile sink in any coordinate space. Sink location in each logical coordinate space is still [2, 1, 0], as also use $\mathrm{K}=1, \mathrm{dv}=3$. If each mobile sink has a different step size $\mathrm{K}$ value, sensor nodes will calculate neighbors' distances to multiple destination references and select route accordingly. Figure. 4 gives us an example of data gathering in multiple coordinate spaces. For node n5, its neighbor node $\mathrm{n} 3$ 's vector distance to $[2,1,0]$ with regard to the black sink is $\sqrt{11}$ and $\sqrt{5}$ to the red sink. While for node $n 4$, its distances are 3 and $\sqrt{5}$ respectively. So either $n 3$ or $n 4$ can be used as the next hop to route to the red sink.

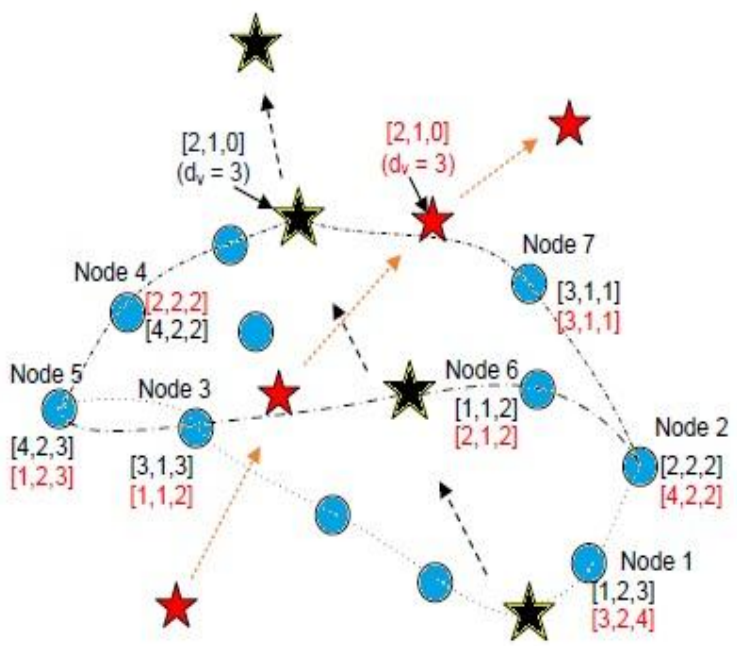

Figure 4. SinkTrail of multiple mobile sinks scenario.

\section{EXPERIMENTAL RESULTS AND SIMULATION}

The proposed protocol is implemented in Network Simulator (NS2) software. In this simulation they have compared the implemented protocol modules i.e.STPOM (SinkTrail protocol with one mobile sink) and STPMM (SinkTrail protocol with multiple mobile sink). The parameters used for comparison between these SinkTrail protocols are packet delivery ratio, control overhead, route length, STD route length, delay, throughput, delay, jitter, dropping ratio, total energy consumption. All these parameters are analyzed with network simulator and their performances are presented in Figure 5 to Figure 7 and the analysis of all these figures is summarized in Table 3. Simulation parameters used are listed 
in Table 2 for quick reference.

Table 2. Simulations parameters.

\begin{tabular}{|c|c|c|c|}
\hline Parametrs & Value & Parametrs & Value \\
\hline $\begin{array}{c}\text { Souce } \\
\text { Type }\end{array}$ & MAC & $\begin{array}{c}\text { Transmissio } \\
\text { n Range }\end{array}$ & $200 \mathrm{~m}$ \\
\hline $\begin{array}{c}\text { No of } \\
\text { Nodes }\end{array}$ & $200,400,600$ & $\begin{array}{c}\text { Simulation } \\
\text { Area }\end{array}$ & $\begin{array}{c}1000 * 1 \\
000\end{array}$ \\
\hline $\begin{array}{c}\text { Simulatio } \\
\text { n Time }\end{array}$ & $200 \mathrm{sec}$ & Node Speed & $20 \mathrm{~m} / \mathrm{s}$ \\
\hline $\begin{array}{c}\text { Packet } \\
\text { Size }\end{array}$ & $2000 \mathrm{bytes}$ & $\begin{array}{c}\text { Initial } \\
\text { Energy }\end{array}$ & $\begin{array}{c}100 \\
\text { joules }\end{array}$ \\
\hline $\begin{array}{c}\text { Routing } \\
\text { Protocol }\end{array}$ & STPOM,STPMM & Pause Time & $00 \mathrm{~ms}$ \\
\hline
\end{tabular}

- Following points are noted from Figure 5 to Figure 12.

- When number of node increases PDR of STPMM is decreases and PDR of STPOM increases as shown in Figure 5. PDR=Total no of packet received/ Total no of packet sent.

- When number of node increases control overhead of STPOM decreases while control overhead of STPMM is increases as shown in Figure 6. This ratio is calculated by comparing the total number of routing packets transmitted during the simulation time to the number of data packets delivered.

- Simulated values of delay represent reliability of routing protocol in the network. As no of nodes increases delay of STPMM increases while delay of STPOM decreases as shown in Figure 7.

- Figure 8 shows when no of nodes increases throughput of STPOM increases while throughput of STPMM decreases.

- Jitter is reduced with time. Jitter is employed to avoid collisions caused by simultaneous transmission by adjacent nodes over the same channel. As the number of packets increased over the same channel, jitter is increased which leads to the loss of data (Figure 9).

The performance of protocol is compared along with presence of multihop, single mobile sink, multiple mobile sink with delay, pdr, control overhead, route length, energy consumption etc. Shown in Table 3.

Following points are noted from Figure 13 to Figure 15.

- When the number of nodes increases PDR of single mobile sink comparatively low than the others shown in Figure 13.

- Total energy consumption based on data packet forwarding cost, routing table maintenance cost, trail message. So when the total no of nodes increases total energy consumption of multiple mobile sink comparatively decreases than others.

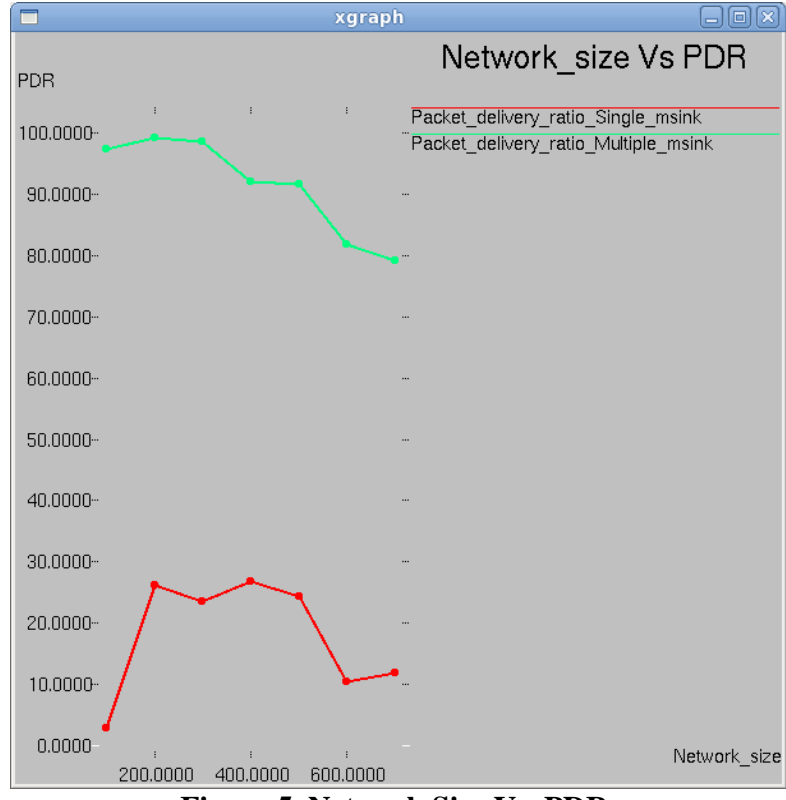

Figure 5. Network Size Vs. PDR.

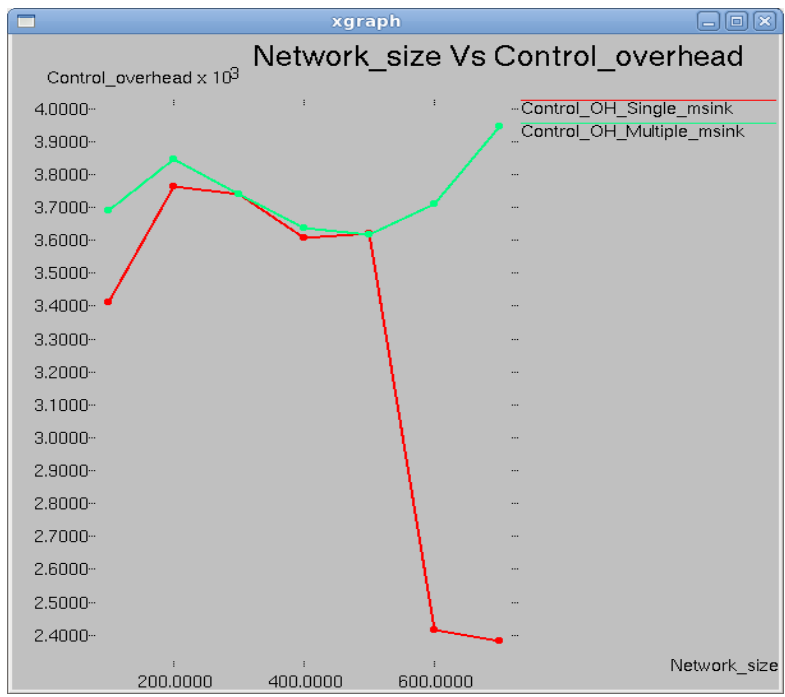

Figure 6.Network size Vs. control overhead

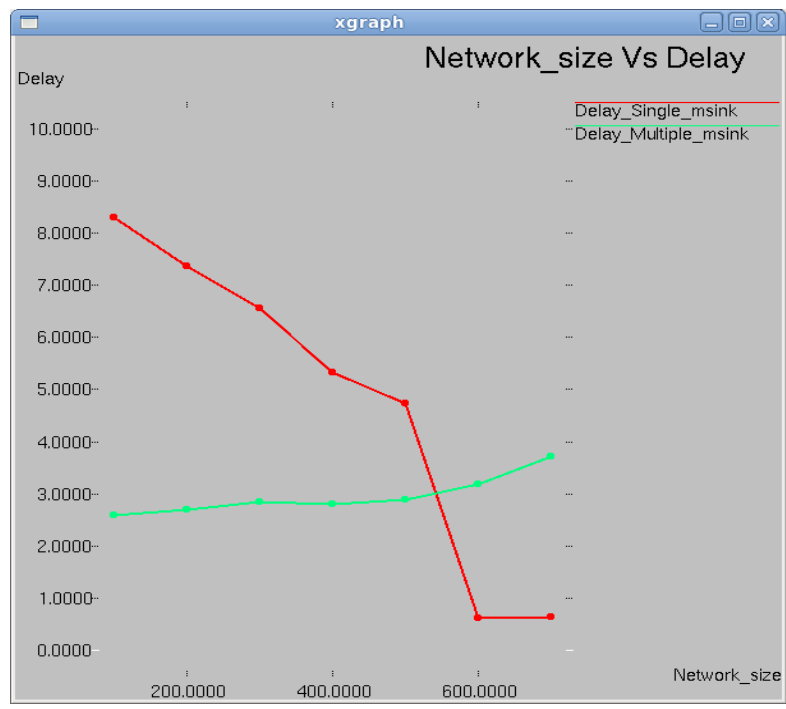

Figure 7.Network size Vs Delay. 


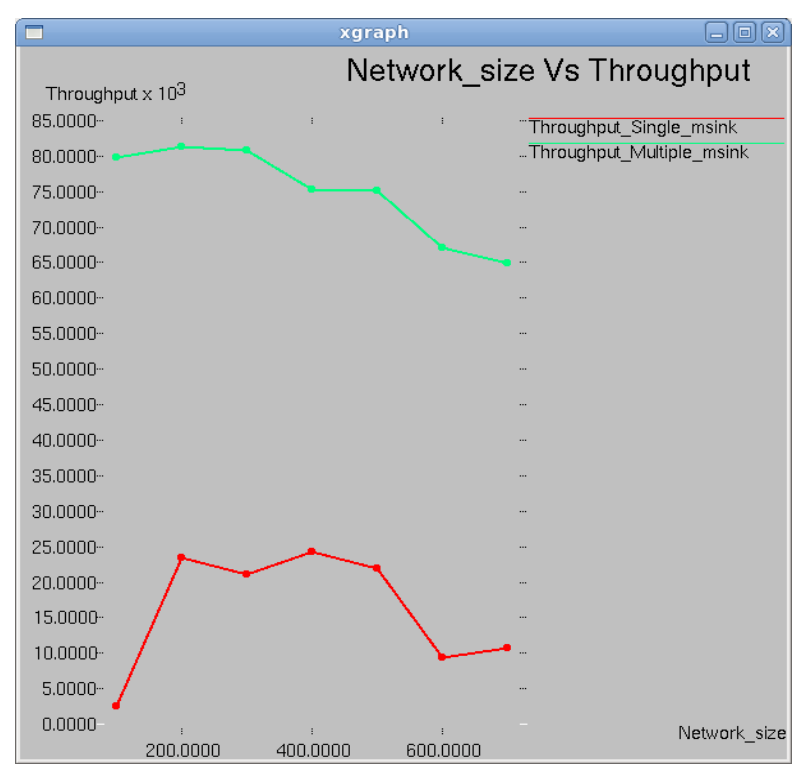

Figure 8.Network size Vs. Throughput.

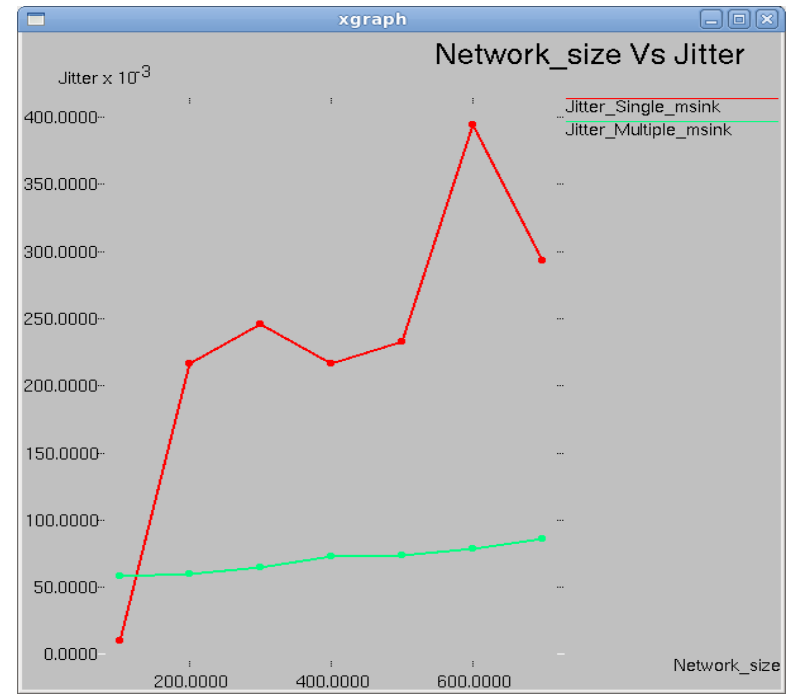

Figure 9.Network size vs. Jitter

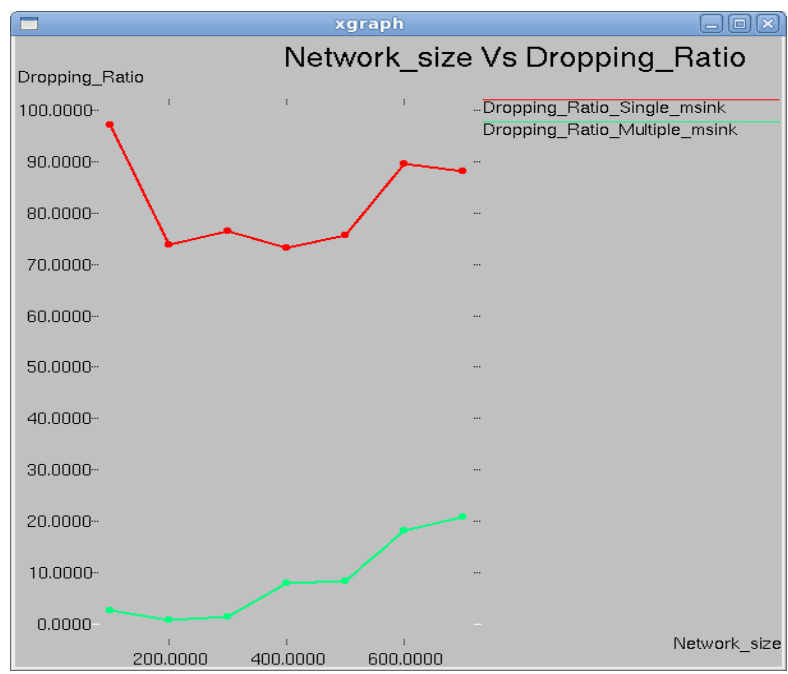

Figure 10.Network Size Vs. Dropping Ratio.

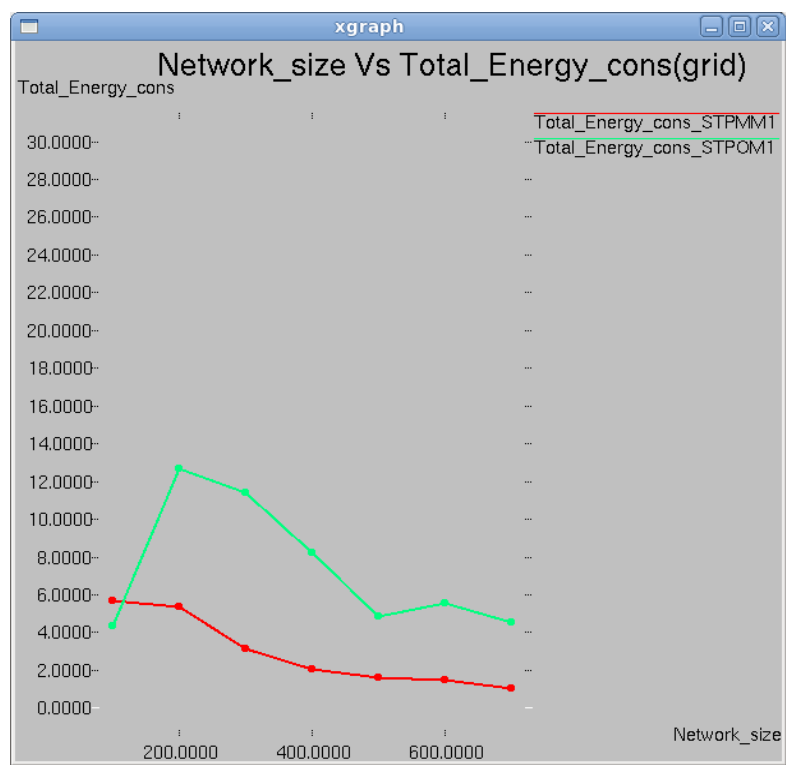

Figure 11.Network Size Vs. Energy consumption.

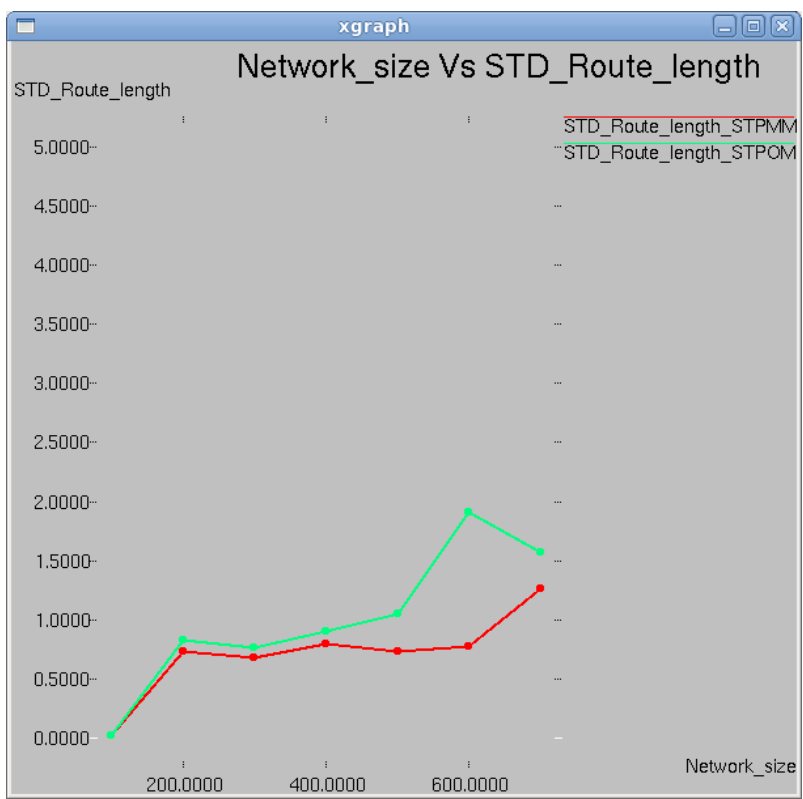

Figure 12.Network Size Vs. STD Route Length.

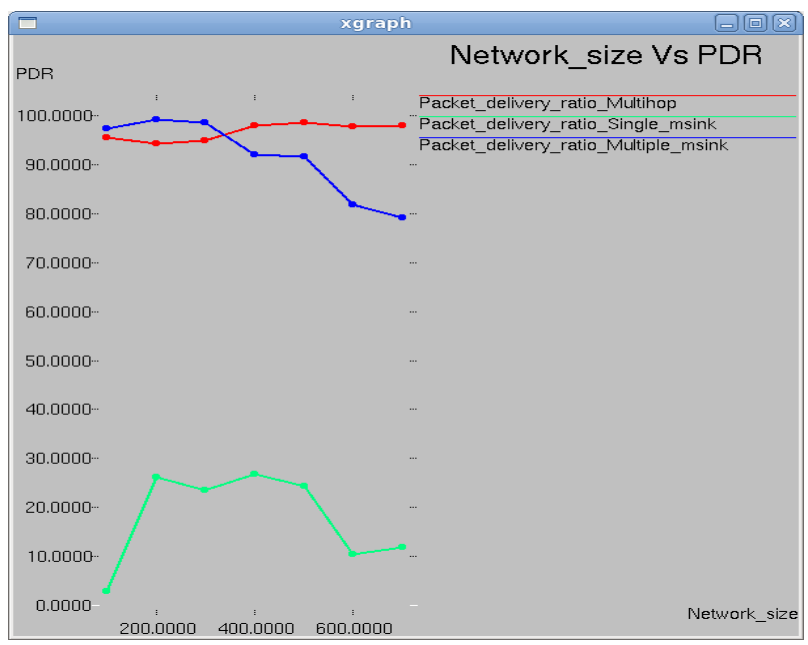

Figure 13.Network Size Vs. PDR. 


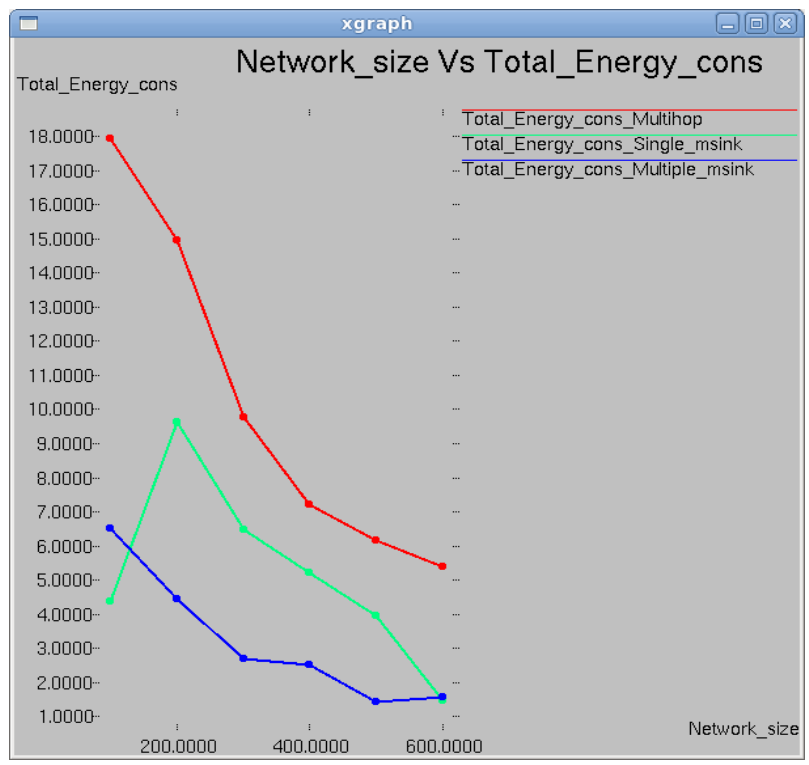

Figure 14.Network Size Vs. Total Energy Consumption.

Table 3: Performance Analysis of Different Parameters with Protocol.

\begin{tabular}{|c|c|c|c|}
\hline Parameter & Multihop & $\begin{array}{c}\text { Single } \\
\text { Mobile } \\
\text { Sink }\end{array}$ & $\begin{array}{c}\text { Multiple } \\
\text { Mobile } \\
\text { Sink }\end{array}$ \\
\hline PDR & Increases & Increases & Decreases \\
\hline Delay & Constants & Decreases & Increases \\
\hline Throughput & Constants & Increases & Decreases \\
\hline Jitter & Constants & Increases & Increases \\
\hline $\begin{array}{c}\text { Energy cons } \\
\text { Dropping } \\
\text { Ratio }\end{array}$ & Decreases & Decreases & Decreases \\
\hline
\end{tabular}

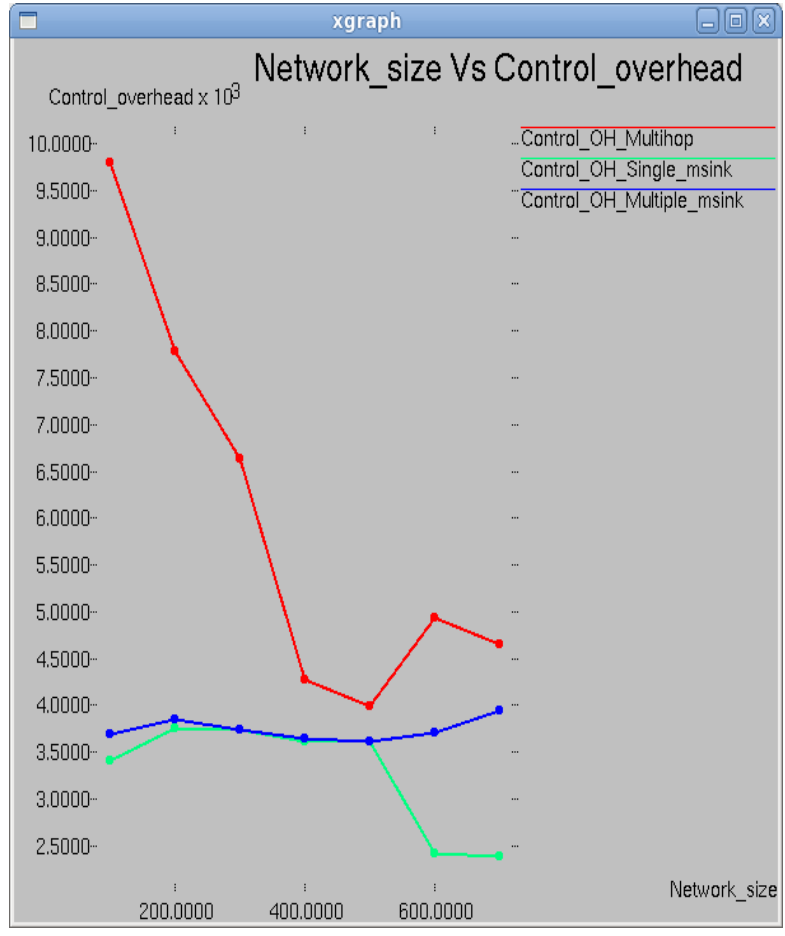

Figure 15.Network Size Vs. Energy consumption.

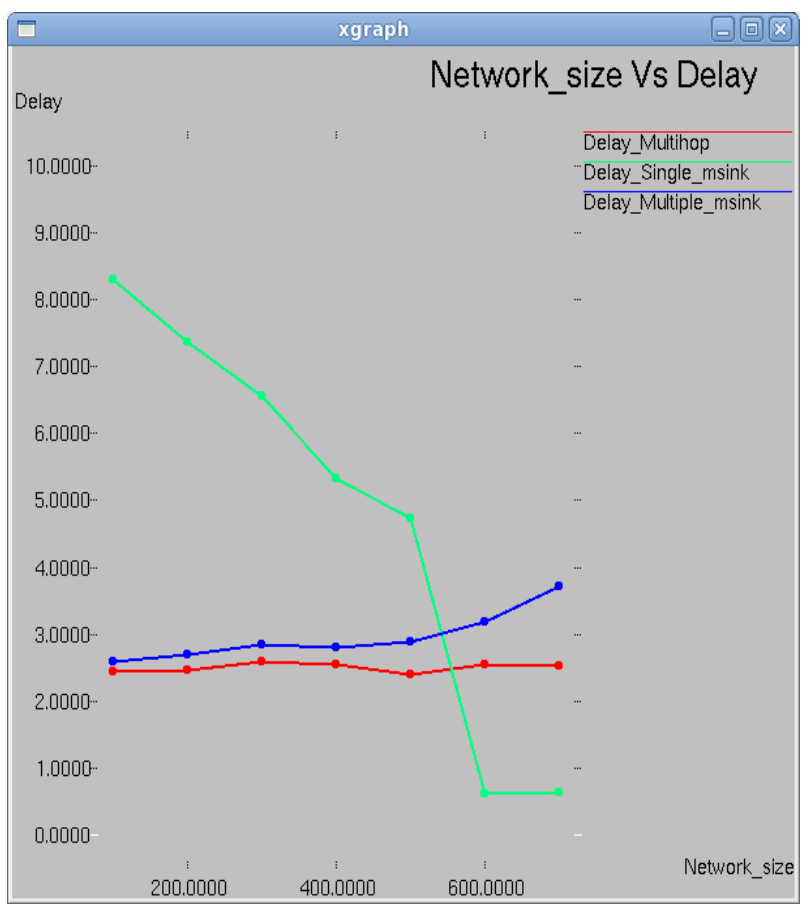

Figure 16.Network Size Vs. Delay.

\section{CONCLUSION}

They proposed the SinkTrail proactive data reporting protocols for energy-efficient data gathering which has low complexity. SinkTrail performs logical coordinates to indicate the distances, and establishes data reporting routes by greedily selecting the shortest path to the destination reference trail point. Also SinkTrail is adequate of locating multiple mobile sinks simultaneously through multiple logical coordinate spaces in the network. Further are some advantages of SinkTrail as follows

- It eliminates the need of special treatments for 
changing field situations.

- SinkTrail is self-adaptable to various sensor field shapes and different moving patterns of mobile sinks.

- It has unique feature of geographical routing without GPS system or predefined landmarks installed.

From the above simulated result they conclude that analyzed energy consumptions of SinkTrail and other representative approaches and validated their analysis through extensive simulations. The simulated results states that SinkTrail finds short data reporting routes and effectively reduces energy consumption.

\section{REFERENCES}

[1]. D. Coffin, D. Van Hook, S. McGarry, and S. Kolek, "Declarative Ad-Hoc Sensor Networking," Proc. SPIE, vol. 4126, p. 109, 2000.

[2]. M. Demirbas, O. Soysal, and A. Tosun, "Data Salmon: A Greedy Mobile Basestation Protocol for Efficient Data Collection in Wireless Sensor Networks," Proc. IEEE Third Int'l Conf. Distributed Computing in Sensor Systems, pp. 267-280, 2007.

[3]. Q. Huang, C. Lu, and G. Roman, "Spatiotemporal Multicast in Sensor Networks," Proc. First ACM Conf. Embedded Networked Sensor Systems (SenSys), pp. 205-217, 2003.

[4]. C. Intanagonwiwat, R. Govindan, and D. Estrin, "Directed Diffusion: A Scalable and Robust Communication Paradigm for Sensor Networks," Proc. MobiCom, pp. 56-67, 2000

[5]. M. Keally, G. Zhou, and G. Xing, "Sidewinder: A Predictive Data Forwarding Protocol for Mobile Wireless Sensor Networks," Proc.IEEE Sixth Ann Comm. Soc. Conf. Sensor, Mesh and Ad Hoc Comm.and Networks (SECON), pp. 1-9, June 2009.

[6]. B. Liu, W. Ke, C. Tsai, and M. Tsai, "Constructing a Message-Pruning Tree with minimum Cost for Tracking Moving Objects in Wireless Sensor Networks is Npcomplete and an Enhanced Data Aggregation Structure," IEEE Trans. Computers, vol. 57, no. 6,pp. 849-863, June 2008

[7]. J. Luo and J.-P. Hubaux, "Joint Mobility and Routing for Lifetime Elongation in Wireless Sensor Networks," Proc. IEEE INFOCOM,vol. 3, 2005.

[8]. M. Ma and Y. Yang, "Data Gathering in Wireless
Sensor Networks with Mobile Collectors," Proc. IEEE Int'l Symp. Parallel and Distributed Processing (IPDPS), pp. 1-9, Apr. 2008.

[9]. T. Park, D. Kim, S. Jang, S. eun Yoo, and Y. Lee, "Energy Efficient and Seamless Data Collection with Mobile Sinks in Massive Sensor Networks," Proc. IEEE Int'l Symp. Parallel and Distributed Processing (IPDPS), pp. 1-8, May 2009.

[10].A.A. Somasundara, A. Ramamoorthy, and M.B. Srivastava,"Mobile Element Scheduling for Efficient Data Collection in Wireless Sensor Networks with Dynamic Deadlines," Proc. IEEE 25th Int'l Real-Time Systems Symp. (RTSS), pp. 296-305, 2004.

[11].O. Soysal and M. Demirbas, "Data Spider: A Resilient Mobile Basestation Protocol for Efficient Data Collection in Wireless Sensor Networks," Proc. Int'l Conf. Distributed Computing in Sensor Systems (DCOSS), 2010

[12].F. Ye, H. Luo, J. Cheng, S. Lu, and L. Zhang, “A Twotier Data Dissemination Model for Large-Scale Wireless Sensor Networks,"Proc. MobiCom, pp. 148-159, 2002.

[13].F. Ye, G. Zhong, S. Lu, and L. Zhang, "Gradient Broadcast: A Robust Data Delivery Protocol for Large Scale Sensor Networks,"Wireless Networks, vol. 11, no. 3, pp. 285-298, 2005.

[14].M. Zhao, M. Ma, and Y. Yang, "Mobile Data Gathering with Space-Division Multiple Access in Wireless Sensor Networks,"Proc. IEEE INFOCOM, pp. 1283-1291, Apr. 2008

[15].M. Zhao, M. Ma, and Y. Yang, "Efficient Data Gathering with Mobile Collectors and Space-Division Multiple Access Technique in Wireless Sensor Networks," IEEE Trans. Computers, vol. 60, no. 3,pp. 400-417, Mar. 2011.

[16].M. Zhao and Y. Yang, "Bounded Relay Hop Mobile Data Gathering in Wireless Sensor Networks," IEEE Trans. Computers,vol. 61, no. 2, pp. 265-277, Feb. 2012.

[17].Xinxin Liu, Han Zhao, Xin Yang, Xiaolin Li, "SinkTrail: A Proactive Data Reporting Protocol for Wireless Sensor Networks," IEEE TRANSACTIONS ON COMPUTERS, VOL. 62, NO. 1, JANUARY 2013.

[18].Parvathy C. A, A. Mahalakshmi," An Energy Efficient Dat Reporting Technique in Wireless Sensor Networks,' International Journal of Engineering Research \& Technology (IJERT), Vol. 3 Issue 1, January - 2014. 